\title{
A design approach for structured controllers for uncertain delay systems grounded in the real structured pseudospectra framework
}

\author{
Francesco Borgioli* Wim Michiels*
}

July 17, 2018

\begin{abstract}
When investigating the stability of delay systems, the role of uncertainties on physical parameters cannot be neglected. Considering linear time-invariant systems and adopting a frequency domain approach, the presence of uncertainties induces a repositioning of the characteristic roots of the system; the pseudospectral abscissa is the largest real part that the eigenvalues can take, and therefore it represents an efficient measure of the stability robustness of a system. In this chapter we consider linear delay systems affected by uncertainties on both the system matrices and the delay terms, and we propose a method to minimize the pseudospectral abscissa as a function of design or controller parameters. With the purpose of a most realistic description of the problem, all the uncertainties affecting the system are considered real-valued, and the approach does not involve any over-bounding of the uncertainty. Moreover, the approach entirely exploits the structure of the delay equation, as well as structure on and potential inter-dependencies among the uncertainties on system matrices. The method supports a system model in terms of DDAEs (Delay Differential Algebraic Equations), which are very flexible as they allow model (sub)systems, controllers, and their interconnections in a systematic way. This approach proves to be very effective, and particularly useful in the design of robust static or dynamic fixed-order controllers for a system of DDEs (Delay Differential Equations) of retarded type. This is illustrated with several numerical examples, including problems where delays are used as controller parameters.
\end{abstract}

Keywords: Time delay, stability robustness, perturbation analysis, uncertain linear systems, pseudospectral abscissa.

\section{Introduction}

The stabilisation of linear time-delay systems is a topic of major concern in control systems theory and a substantial amount of results have contributed to develop and partially solve this problem; without being exhaustive, possible approaches for stability analysis and stabilization are the use of Lyapunov-Krasovskii functionals and LMI conditions (see for instance $[5,25,27]$ ), the direct eigenvalue optimization approach ([28]), the continuous pole placement method ([17]) and the use of prediction based controllers (see [14] and references therein, $[24,20])$. We refer the reader interested in a general overview on the many different stabilisation methods to the monographs [22], [7] and [21]. In this work we consider problems where the closed loop system takes the form

$$
E \dot{x}(t)=A_{0}(p) x(t)+\sum_{i=1}^{m} A_{i}(p) x\left(t-\tau_{i}(p)\right),
$$

where $E \in \mathbb{R}^{n \times n}$ is allowed to be a singular matrix (see Section 2 for a detailed description of the assumptions), $x(t) \in \mathbb{R}^{n}$ is the state variable, $A_{0}(p), \ldots, A_{m}(p) \in \mathbb{R}^{n \times n}, 0<\tau_{1}(p)<\cdots<\tau_{m}(p)$ are the delay terms, and $p \in \mathbb{R}^{n_{p}}$ is a vector of design or controller parameters. Note that this general form also allows to address problems where delays are used as controller parameters.

Most of the times a controller is implemented in order to make a system asymptotically stable; using a frequency approach, this means to push all the roots of its associated characteristic equation to the open lefthalf of the complex plane, or equivalently, to have a negative spectral abscissa, i.e. the real part of the rightmost

*Department of Computer Science, Katholieke Universiteit Leuven, Celestijnenlaan 200A, 3001 Heverlee, Belgium (\{firstname.lastname\}@cs.kuleuven.be). 
eigenvalue in the spectrum. Minimizing the spectral abscissa w.r.t some control or design parameters, as done in [28], is therefore a simple and effective method to stabilize a time-delay system. However, the spectral abscissa is not a good measure for the stability robustness: in real-life applications uncertainties on system parameters are always present and potentially affect the system's stability; in particular, they affect the spectrum of the system, generating a shift of its eigenvalues in the complex plane.

In real applications, we can assume to have an upper bound $\varepsilon$ on the size of the uncertainties affecting our system of interest: the $\varepsilon$-pseudospectrum is then defined as the region in the complex plane to which eigenvalues can be moved when the system is subject to some $\varepsilon$-bounded uncertainties; the $\varepsilon$-pseudospectral abscissa is then the supremum of the real parts of the points in the $\varepsilon$-pseudospectrum, and it constitutes a bound on the asymptotic growth rate of the solution of an uncertain system, which is uniform over all $\varepsilon$-bounded uncertainties. As a consequence, a negative pseudospectral abscissa guarantees the robustness of the asymptotic stability of system (1.1) against any $\varepsilon$-bounded uncertainty on the model; in this chapter we propose a method to robustly stabilize system (1.1) via the minimization of its pseudospectral abscissa w.r.t. a fixed set of parameters $p$.

In the literature, important distinctions are made regarding the perturbations of the system matrices: perturbations can be considered either complex-valued or real-valued, where the latter ones are certainly more realistic from an application point of view. Perturbations can also be distinguished between structured and unstructured, where the former ones allow the perturbation of any specific submatrix, e.g. a single coefficient or a block. Among the many algorithms for the computation of the pseudospectral abscissa, we refer the reader to [8] and [19]; the former exploits unstructured real-valued perturbations of a standard eigenvalue problem, whereas the latter applies to nonlinear eigenvalue problems (e.g. polynomial and delay eigenvalue problems) whose matrices are perturbed by unstructured complex-valued matrices. In [1], we inherited the real-valued feature from the former and the nonlinearity structure from the latter: moreover, we simultaneously considered real-valued uncertainties on the delay terms and real-valued structured uncertainties on the system matrices. In addition to this method, as main contribution in this chapter we take into account the potential dependency between the uncertainties perturbing the nominal matrices $A_{i}$ : this has a major relevance for those uncertain system parameters which are present in more than one matrix $A_{i}$ and will be motivated by an application from machine tool vibrations.

An original, distinctive feature of the overall approach in this chapter is that robustness of stability, expressed in terms of negative pseudospectral abscissa, is assessed by solving an eigenvalue optimization problem where the real part of the rightmost eigenvalue is maximized as a function of the allowable perturbations. First, this setting allows an exact description of a broad class of uncertainties. As a matter of fact, the special structure of the delay system, the property that uncertain parameters are real valued, possible interdependencies between perturbations, and a nonlinear dependence on uncertain data (uncertainties affecting delay values too) can be easily exploited. Particularly the latter is very difficult within the standard approaches in robust control, which are grounded in structured singular value characterizations (see for instance [10, 18]). Second, due to use of rightmost eigenvalue computations and the exploitation of the structure on the uncertainty, the obtained robust stability conditions are necessary and sufficient, in contrast to many approaches grounded in Lyapunov's second method ([3]) and resulting in potentially very conservative criteria, e.g. LMI based ones (see [6] and [4] for a tutorial). Third, we adopt a system description in terms of DDAEs (1.1), which allow to systematically model interconnections of subsystems (including interconnections between systems and controllers).

Furthermore, in this chapter we make the leap from robust stability analysis to the design of robustly stabilizing controllers. The distinctive feature of the adopted approach is that this is done by minimizing the pseudospectral abscissa (in such a way that the optimization problem behind the overall approach can be seen as a min-max (saddle point) problem); since the pseudospectral abscissa is by definition a continuous but only almost everywhere differentiable function, the optimization is performed using the HANSO method (Hybrid Algorithm for Nonsmooth Optimization) introduced in [15] (see website indicated in [23] for a detailed description). An additional advantage is that structured controllers can be designed, e.g. low order controllers that are easy to implement (note that within a Lyapunov framework the design of reduced order controllers would give to complicated bilinear matrix inequalities). Some preliminary results in this direction, without taking into account interdependencies between perturbations, are reported in [2].

The chapter is structured as follows: in Section 2 we formally introduce the uncertainties on system (1.1), the corresponding delay eigenvalue problem and the pseudospectral approach used to investigate the stability robustness; in Section 3 we review and extend the method to compute the pseudospectral abscissa introduced 
in [1]; in Section 4 we briefly describe the smoothness properties of the pseudospectral abscissa and compute the gradient of the pseudospectral abscissa w.r.t. design or controller parameters employed as optimization variables; in Section 5 we show the applicability of this method to linear delay systems with static or dynamic controllers and present some numerical experiments; conclusions are finally presented in Section 6.

\section{Robust stability of an uncertain system: a pseudospectral approach}

In Sections 2 and 3 we model the uncertainty on system (1.1), define and present an algorithm for the pseudospectral abscissa computation. Since we consider the set of controller parameters $p$ as fixed, in these two sections we suppress the dependence of $A_{i}$ on $p$ in the notations (for the optimization of the pseudospectral abscissa as a function of $p$ we refer to Section 4).

We consider system (1.1) and we want to include real-valued uncertainties on the matrices $A_{i}$ and on the delay terms $\tau_{i}$; uncertainties affecting matrices $A_{i}$ are also allowed to be structured, and we admit an interdependency between them. Therefore, we assume the existence of $K$ independent uncertainties $\delta A_{j}$, where each $\delta A_{j}$ can perturb one or more nominal matrix $A_{i}$, and the existence of $m$ uncertainties $\delta \tau_{i}$. Introducing these uncertainties in system (1.1) we have

$$
E \dot{x}(t)=\left(A_{0}+\sum_{j=1}^{K} B_{i, j} \delta A_{j} C_{i, j}\right) x(t)+\sum_{i=1}^{m}\left(A_{i}+\sum_{j=1}^{K} B_{i, j} \delta A_{j} C_{i, j}\right) x\left(t-\left(\tau_{i}+\delta \tau_{i}\right)\right),
$$

where $\delta A_{j} \in \mathbb{R}^{p_{j} \times q_{j}}, \delta \tau_{i} \in \mathbb{R}$ and is such that $\left|\delta \tau_{i}\right|<\tau_{i}$; each nominal matrix $A_{i}$ can be perturbed by one or more uncertainties $\delta A_{j}$, whose structures (and weights) are defined by auxiliary matrices $B_{i, j}$ and $C_{i, j}$. Matrices $B_{i, j}$ and $C_{i, j}$ are real-valued and their dimensions depend on the dimension of the associated uncertainty $\delta A_{j}$ : as a consequence, for each $\delta A_{j} \in \mathbb{R}^{p_{j} \times q_{j}}$, we have $B_{i, j} \in \mathbb{R}^{n \times p_{j}}$ and $C_{i, j} \in \mathbb{R}^{q_{j} \times n}$ for all $i=0, \ldots, m, j=1, \ldots, K$. Obviously, if matrix $A_{i}$ is not affected by uncertainty $\delta A_{j}$, we assume $B_{i, j}=0$ or $C_{i, j}=0$. In the following, with the purpose of simplifying the notation, we will indicate with $\mathbb{R}^{*}=\left(\mathbb{R}^{p_{1} \times q_{1}} \times \cdots \times \mathbb{R}^{p_{K} \times q_{K}}\right)$ the domain of the tuple of uncertainties $\left(\delta A_{1}, \ldots, \delta A_{K}\right)$.

We here introduce the delay eigenvalue problem (DEP) associated with the system of perturbed equations

$$
M(\lambda) y:=\left(\lambda E-\sum_{i=0}^{m}\left(A_{i}+\sum_{j=1}^{K} B_{i, j} \delta A_{j} C_{i, j}\right) e^{-\lambda\left(\tau_{i}+\delta \tau_{i}\right)}\right) y=0,
$$

where $\lambda \in \mathbb{C}$ and $y \in \mathbb{C}^{n}$. In addition, with the purpose of a simpler notation, we make the system more compact by adopting $\tau_{0}=0, \delta \tau_{0}=0$.

The general DDAE models (1.1) and (2.1) may arise as the feedback interconnection of a plant model with a controller with a fixed-order or structure, where $p$ in (1.1) is the parameterization of this controller. We here give two examples to explain the definition of the uncertainties and the benefits derived from a DDAE formulation; the same examples will be considered again in Section 5. In the first one, we show a classic case of an uncertain system with static feedback controller that can be recast using DDAEs; in the second one, we consider a model for a rotating cutting machine affected by uncertainties.

Example 2.1. Consider the system with uncertainties

$$
\left\{\begin{array}{l}
\dot{x}(t)=(A+\delta A) x(t)+(B+\delta B) u(t-(\tau+\delta \tau)) \\
y(t)=(C+\delta C) x(t)
\end{array}\right.
$$

with static controller

$$
u(t)=K y(t) .
$$


Defining the new state variable $\boldsymbol{\xi}:=(x, y, u)^{T}$, the system can be recast as follows

$$
\begin{aligned}
& \underbrace{\left[\begin{array}{lll}
I & 0 & 0 \\
0 & 0 & 0 \\
0 & 0 & 0
\end{array}\right]}_{:=\boldsymbol{E}} \dot{\boldsymbol{\xi}}(t)=(\underbrace{\left[\begin{array}{ccc}
A & 0 & 0 \\
C & -I & 0 \\
0 & K & -I
\end{array}\right]}_{:=\boldsymbol{A}_{0}}+\underbrace{\left[\begin{array}{l}
I \\
0 \\
0
\end{array}\right] \delta A\left[\begin{array}{l}
I \\
0 \\
0
\end{array}\right]^{T}}_{:=\boldsymbol{B}_{0,1} \boldsymbol{\delta} \boldsymbol{A}_{1} \boldsymbol{C}_{\mathbf{0}, 1}}+\underbrace{\left.\left[\begin{array}{c}
0 \\
I \\
0
\end{array}\right] \delta C\left[\begin{array}{l}
I \\
0 \\
0
\end{array}\right]^{T}\right)}_{:=\boldsymbol{B}_{0,2} \boldsymbol{\delta} \boldsymbol{\boldsymbol { A } _ { \boldsymbol { 2 } } \boldsymbol { C } _ { \mathbf { 0 } , 2 }}} \boldsymbol{\xi}(t)+ \\
& +(\underbrace{\left[\begin{array}{lll}
0 & 0 & B \\
0 & 0 & 0 \\
0 & 0 & 0
\end{array}\right]}_{:=\boldsymbol{A}_{1}}+\underbrace{\left[\begin{array}{l}
I \\
0 \\
0
\end{array}\right] \delta B\left[\begin{array}{l}
0 \\
0 \\
I
\end{array}\right]}_{:=\boldsymbol{B}_{1,3} \delta \boldsymbol{A}_{3} C_{1,3}}) \boldsymbol{\xi}(t-(\tau+\delta \tau)) \text {. }
\end{aligned}
$$

In the last equation we reformulated the original problem in form of DDAEs with perturbations as in (2.1), where $p$ in (1.1) is the vectorization of the controller parameters in $K$. Note that the elimination of input $u$ would lead to a nonlinear dependence of the system matrices on the uncertainty: as a matter of fact, if one substitutes control law (2.4) in the system of DDAE (2.3), then the representation (2.1) is not valid anymore, since the uncertainty $\delta B$ cannot be decoupled from the controller parameters in $\mathrm{K}$; the adopted reformulation in a DDAE framework then allows us to solve this problem. More in general, thanks to the introduction of a slack variable, we are able to handle any polynomial dependence on the uncertainties within a DDAE framework.

Furthermore, original perturbations $\delta A, \delta C$ are treated as two independent perturbations (renamed $\delta A_{1}$ and $\delta A_{2}$ respectively) affecting matrix $A_{0}$ of the new system of DDAE; the original $\delta B$ is another independent perturbation affecting matrix $A_{1}$ of the new system.

Example 2.2. We consider a simple model for a turning process as presented in [11] and [12]

$$
\ddot{x}(t)+2 \xi \omega \dot{x}(t)+\omega^{2} x(t)=\frac{k}{m}(x(t-\tau)-x(t)),
$$

whose corresponding DEP reads

$$
\left(\lambda E-A_{0}-A_{1} e^{-\lambda \tau}\right) y=0,
$$

with

$$
E=\left[\begin{array}{ll}
1 & 0 \\
0 & 1
\end{array}\right], A_{0}=\left[\begin{array}{cr}
0 & 1 \\
-\omega^{2}-\frac{k}{m} & -2 \xi \omega
\end{array}\right], A_{1}=\left[\begin{array}{cc}
0 & 0 \\
\frac{k}{m} & 0
\end{array}\right],
$$

and where $\omega$ is the natural frequency, $\xi$ the damping ratio, $m$ the modal mass and $k$ the cutting force coefficient. Considering system (1.1), we then have $\tau_{1}=\tau$.

Let us now assume that parameters $k$ and $\xi$ are respectively affected by uncertainties $\delta k=\delta A_{1}$ and $\delta \xi=\delta A_{2}$, and that delay term $\tau_{1}$ also admits a perturbation $\delta \tau_{1}$. Then, we obtain the perturbed DEP

$$
M(\lambda)=\lambda E-\left(A_{0}+B_{0,1} \delta k C_{0,1}+B_{0,2} \delta \xi C_{0,2}\right)-\left(A_{1}+B_{1,1} \delta k C_{1,1}\right) e^{-\lambda\left(\tau_{1}+\delta \tau_{1}\right)},
$$

where

$$
B_{0,1}=-B_{1,1}=\left[\begin{array}{c}
0 \\
-\frac{1}{m}
\end{array}\right], C_{0,1}=C_{1,1}=\left[\begin{array}{l}
1 \\
0
\end{array}\right]^{T}, B_{0,2}=\left[\begin{array}{c}
0 \\
-2 \omega
\end{array}\right], C_{0,2}=\left[\begin{array}{l}
0 \\
1
\end{array}\right]^{T} .
$$

In this case, perturbation $\delta k=\delta A_{1}$ thus affects both nominal matrices $A_{0}$ and $A_{1}$. The algorithm presented here to compute the pseudospectral abscissa, which generalizes the one introduced in [2], enables us to impose that the perturbation $\delta A_{1}$ affecting both nominal matrices $A_{0}$ and $A_{1}$ stems from the same uncertain parameter $k$.

Now we need to define an overall scalar measure of the uncertainties affecting our system of interest. We first define the set of all the uncertainties as

$$
\Delta:=(\Delta A, \Delta \tau)=\left(\delta A_{1}, \ldots, \delta A_{K}, \delta \tau_{1}, \ldots, \delta \tau_{m}\right),
$$

and then, after introducing weights $v_{i} \in \mathbb{R}^{+} \cup\{+\infty\}$, we define the global norm

$$
\|\Delta\|:=\left\|\left[\begin{array}{llllll}
\left\|\delta A_{1}\right\|_{F} & \ldots & \left\|\delta A_{K}\right\|_{F} & v_{1}\left|\delta \tau_{1}\right| & \ldots & v_{m}\left|\delta \tau_{m}\right|
\end{array}\right]^{T}\right\|_{\infty} .
$$


where $\|\cdot\|_{F}$ indicates the matrix Frobenius norm. Given this definition, a set of perturbations $\Delta$ is $\varepsilon$-bounded if and only if

$$
\left\|\delta A_{j}\right\|_{F} \leq \varepsilon, \quad\left|\delta \tau_{i}\right| \leq \frac{\varepsilon}{v_{i}}, \quad j=1, \ldots, K, i=1, \ldots, m .
$$

Moreover, if a weight $v_{i}=+\infty$ then the corresponding delay term $\tau_{i}$ is assumed free from uncertainty. It is worth to highlight that definition (2.8) allows bounds on different perturbations to be specified independently on each other; in this sense, it is possible to treat the case where two or more independent uncertainties are simultaneously set equal to their maximum allowed value. Equivalently, the space of $\varepsilon$-bounded perturbations can be represented as an hypercube rather than an hypersphere.

At this point we can define the real-valued, structured $\varepsilon$-pseudospectrum as the following set

$$
\Lambda_{\varepsilon}:=\bigcup_{\substack{\Delta \in \mathbb{R}^{*} \times \mathbb{R}^{m} \\\|\Delta\|_{\text {glob }} \leq \varepsilon}}\{\lambda \in \mathbb{C}: \operatorname{det} M(\lambda)=0\},
$$

and the $\varepsilon$-pseudospectral abscissa function $\alpha_{\varepsilon}$ as

$$
\alpha_{\varepsilon}:=\sup \left\{\Re(\lambda): \lambda \in \Lambda_{\varepsilon}\right\} .
$$

In this chapter we will always deal with DDAEs with retarded dynamics, which means in particular that the spectral abscissa of a DEP is always continuous with respect to small variations in the system matrices and in the delay terms; as a consequence, in this case the pseudospectral abscissa can be simply reduced to a maximum function.

We conclude the section presenting the adopted assumptions that guarantee our perturbed DEP (2.2) to have retarded dynamics. Considering system (1.1), let $\operatorname{rank}(E)=n-\nu$ and let $U, V \in \mathbb{R}^{n \times \nu}$ be respectively a (minimal) basis for the left and the right null space of $E$. Then, if we define

$$
\mathbf{U}=\left[U^{\perp} U\right], \mathbf{V}=\left[V^{\perp} V\right] \text { and } x=\mathbf{V}\left[\begin{array}{ll}
x_{1}^{T} & x_{2}^{T}
\end{array}\right]^{T},
$$

system (1.1) can be rewritten as follows

$$
\left\{\begin{array}{l}
E^{(11)} \dot{x_{1}}(t)=\sum_{i=0}^{m} A_{i}^{(11)} x_{1}\left(t-\tau_{i}\right)+\sum_{i=0}^{m} A_{i}^{(12)} x_{2}\left(t-\tau_{i}\right), \\
0=A_{0}^{(22)} x_{2}(t)+\sum_{i=1}^{m} A_{i}^{(22)} x_{2}\left(t-\tau_{i}\right)+\sum_{i=0}^{m} A_{i}^{(21)} x_{1}\left(t-\tau_{i}\right),
\end{array}\right.
$$

where $E^{(11)}=U^{\perp^{T}} E V^{\perp}$, and

$$
\begin{array}{ll}
A_{i}^{(11)}=U^{\perp^{T}} A_{i} V^{\perp}, & A_{i}^{(12)}=U^{\perp^{T}} A_{i} V \\
A_{i}^{(21)}=U^{T} A_{i} V^{\perp}, & A_{i}^{(22)}=U^{T} A_{i} V, \quad i=0, \ldots, m .
\end{array}
$$

We refer to [16], from which we take over the following assumption made throughout the paper.

Assumption 2.1. The matrix $U^{T} A_{0} V$ is nonsingular.

With this assumption, the reformulation of DDAEs as a set of delay differential equations of retarded type coupled with a set of delay difference equations is well-posed and semi-explicit (differentiation index equal to one).

The delay difference equations may induce in the spectrum of DDAEs chains of eigenvalues whose imaginary part tend to infinity while the real part has a finite limit (note that neutral systems can be reformulated as DDAEs); moreover, this limit may be discontinuous with respect to small perturbations of the delay values. Since in these paper we also deal with uncertainties on the delay terms, it is desirable to have continuity of the spectral abscissa w.r.t. delay terms. This is achieved by making the following assumption, which implies that the system has retarded dynamics, and that this is preserved in the presence of perturbations.

Assumption 2.2. Matrices $U^{T} A_{i} V=0$ for $i=1, \ldots, m$. Moreover, for each $(i, j)$ with $i=1, \ldots, m, j=$ $1, \ldots, K$ it must hold that either $U^{T} B_{i, j}=0$ or $C_{i, j} V=0$.

Essentially, by introducing this assumption we eliminate the aforementioned chains of characteristic roots: we refer the reader to [16] for a detailed description of these assumptions. 


\section{Computation of the pseudospectral abscissa}

In this section we illustrate the algorithm for the computation of the pseudospectral abscissa for (2.9), where we summarize the main steps from [2]. As previously mentioned, the novelty here introduced is the potential interdependency among the uncertainties $\delta A_{j}$. We first include the following lemma, which provides a formula for the derivative of an eigenvalue w.r.t. any parameter of the eigenvalue problem; this formula will be widely exploited in this and in the following section, and we refer the reader interested in a proof to Lemma 2.7 in $[26]$.

Lemma 3.1. Let $F(\lambda, \theta): \mathbb{C} \times \mathbb{C}^{d} \longrightarrow \mathbb{C}^{n \times n}$ be continuously differentiable, with $\lambda$ a simple eigenvalue whose corresponding left and right eigenvectors with unit norm are $x$ and $y$, and $\theta$ a set of parameters, then

$$
\frac{\partial \lambda}{\partial \theta_{i}}=-\frac{x^{*} \frac{\partial F(\lambda, \theta)}{\partial \theta_{i}} y}{x^{*} \frac{\partial F(\lambda, \theta)}{\partial \lambda} y}, \quad i=1, \ldots, d
$$

where $x^{*}$ is the conjugate transpose vector of $x$.

The core idea that we implement is to find the maximum real part among the points in the pseudospectrum by taking steps in the direction of the (scaled) gradient of the spectral abscissa of the perturbed DEPs in the space of perturbations. In practice, indicating with $\lambda(\Delta)$ the rightmost eigenvalue of the DEP (2.2) perturbed with $\Delta$, we want to solve the following optimization problem

$$
\begin{array}{cl}
\max & \Re \lambda(\Delta) \\
\text { s.t. } & \Delta \in \mathbb{R}^{*} \times \mathbb{R}^{m}, \\
& \|\Delta\|_{\text {glob }} \leq \varepsilon
\end{array}
$$

The next fundamental theoretical result provides a characterization of the optimal perturbations, namely the perturbations that generate the globally rightmost point in the pseudospectrum, and thus allows us to sensibly reduce the search space of the problem; in particular, it demonstrates that the optimal perturbations affecting the system matrices are always low-rank. Before we state the theorem, we define $k_{j}$ as the number of nominal matrices $A_{i}$ affected by uncertainty $\delta A_{j}$.

Theorem 3.2. Let $\lambda_{\mathrm{RM}}$ be the globally rightmost point of the structured $\varepsilon$-pseudospectrum (2.9) for some optimal $\varepsilon$-bounded perturbation $\Delta=(\Delta A, \Delta \tau)$ and assume it is a simple eigenvalue. Then

(i) There always exists a set of $\varepsilon$-perturbations $\widetilde{\Delta A}=\left(\widetilde{\delta A_{1}}, \ldots, \widetilde{\delta A_{K}}\right)$, such that for each $j=1, \ldots, K$

$$
\operatorname{rank}\left(\widetilde{\delta A_{j}}\right) \leq \rho_{j}^{*}:=\min \left(2 k_{j}, p_{j}, q_{j}\right)
$$

and for which the rightmost eigenvalue is $\lambda_{\mathrm{RM}}$;

(ii) Let $x, y$ be respectively the left and the right eigenvectors (with unitary norms) of $\lambda_{\mathrm{RM}}$ and such that

$$
\xi:=-x\left(E+\sum_{i=0}^{m}\left(A_{i}+\sum_{j=1}^{K} B_{i, j} \delta A_{j} C_{i, j}\right)\left(\tau_{i}+\delta \tau_{i}\right) e^{-\lambda_{\mathrm{RM}}\left(\tau_{i}+\delta \tau_{i}\right)}\right) y>0
$$

and let us define

$$
X=[\mathfrak{R}(x) \mathfrak{I}(x)], \quad Y=[\mathfrak{R}(y) \mathfrak{I}(y)],
$$

and

$$
\Gamma_{0}=\left[\begin{array}{ll}
1 & 0 \\
0 & 1
\end{array}\right], \quad \Gamma_{i}=\left[\begin{array}{cr}
\mathfrak{R}\left(e^{-\left(\tau_{i}+\delta \tau_{i}\right) \lambda_{\mathrm{RM}}}\right) & -\mathfrak{I}\left(e^{-\left(\tau_{i}+\delta \tau_{i}\right) \lambda_{\mathrm{RM}}}\right) \\
\mathfrak{I}\left(e^{-\left(\tau_{i}+\delta \tau_{i}\right) \lambda_{\mathrm{RM}}}\right) & \mathfrak{R}\left(e^{-\left(\tau_{i}+\delta \tau_{i}\right) \lambda_{\mathrm{RM}}}\right)
\end{array}\right], \quad \text { for } i=1, \ldots m .
$$

Now, for each $j=1, \ldots, K$, matrix $\sum_{i=0}^{m} B_{i, j}^{T} X \Gamma_{i} Y^{T} C_{i, j}^{T}$ can be either zero or nonzero. In the latter case the optimal perturbation is

$$
\delta A_{j}=-\varepsilon \frac{\sum_{i=0}^{m} B_{i, j}^{T} X \Gamma_{i} Y^{T} C_{i, j}^{T}}{\left\|\sum_{i=0}^{m} B_{i, j}^{T} X \Gamma_{i} Y^{T} C_{i, j}^{T}\right\|_{F}} .
$$


(iii) Let $x, y$ be defined as before, then

$$
\frac{\partial \Re\left(\lambda_{\mathrm{RM}}\right)}{\partial \delta \tau_{i}}=\frac{1}{\xi} \mathfrak{R}\left(x^{*}\left(A_{i}+\sum_{j=1}^{K} B_{i, j} \delta A_{j} C_{i, j}\right) \lambda_{\mathrm{RM}} e^{-\lambda_{\mathrm{RM}}\left(\tau_{i}+\delta \tau_{i}\right)} y\right)
$$

can also be either zero or nonzero. If it is nonzero, then the optimal time-delay perturbation is such that $v_{i}\left|\delta \tau_{i}\right|=\varepsilon$.

Proof. ( $i$ ) Suppose without loss of generality that the delay terms are free from uncertainties. Moreover, in order to simplify the notation, let us also suppose without loss of generality that each uncertainty $\delta A_{j}$ perturbs the first $k_{j}$ nominal matrices.

It is trivial to see that the relation $\operatorname{rank}\left(\delta A_{j}\right) \leq \min \left\{p_{j}, q_{j}\right\}$ always holds. Let us now also assume that $2 k_{j} \leq \min \left\{p_{j}, q_{j}\right\}$, and thus that $\rho_{j}^{*}=2 k_{j}$. For each $j=1, \ldots, K$, we let $r_{j}^{*} \leq \rho_{j}^{*}$ be the rank of the space spanned by vectors in $Z:=\left\{\mathfrak{R}\left(C_{1, j} y\right), \mathfrak{I}\left(C_{1, j} y\right), \ldots, \mathfrak{R}\left(C_{k_{j}, j} y\right), \mathfrak{I}\left(C_{k_{j}, j} y\right)\right\}$; then we define a matrix $H_{j}$ such that the first $r_{j}^{*}$ columns' span includes the space spanned by vectors in $Z$, and the other columns complete the basis of $\mathbb{R}^{q_{j} \times q_{j}}$, so that $H_{j}$ is orthogonal. Thus, we can write $\delta A_{j}=\widehat{\delta A_{j}} H_{j}^{T}, j=1, \ldots, K$. With this factorization,

$$
\begin{aligned}
0 & =\left(\lambda_{\mathrm{RM}} E-\sum_{i=0}^{m}\left(A_{i}+\sum_{j=1}^{K} B_{i, j} \delta A_{j} C_{i, j}\right) e^{-\lambda_{\mathrm{RM}} \tau_{i}}\right) y \\
& =\left(\lambda_{\mathrm{RM}} E-\sum_{i=0}^{m} A_{i} e^{-\lambda_{\mathrm{RM}} \tau_{i}}\right) y-\sum_{i=0}^{m} \sum_{j=1}^{K} B_{i, j} \widehat{\delta A_{j}} H_{j}^{T} e^{-\lambda_{\mathrm{RM} \tau_{i}}\left(\mathfrak{R}\left(C_{i, j} y\right)+j \Im\left(C_{i, j} y\right)\right)} \\
& =\left(\begin{array}{c}
\left\langle H_{j}^{(1)}, \mathfrak{R}\left(C_{i, j} y\right)+j \mathfrak{I}\left(C_{i, j} y\right)\right\rangle \\
\vdots \\
\left.\lambda_{\mathrm{RM}} E-\sum_{i=0}^{m} A_{i} e^{-\lambda_{\mathrm{RM}} \tau_{i}}\right) y-\sum_{i=0}^{m} \sum_{j=1}^{K} B_{i, j} \widehat{\delta A_{j}} e^{-\lambda_{\mathrm{RM}} \tau_{i}}
\end{array}\left(\begin{array}{c}
\left\langle H_{i}^{\left(r_{j}^{*}\right)}, \mathfrak{R}\left(C_{i, j} y\right)+j \Im\left(C_{i, j} y\right)\right\rangle \\
0 \\
\vdots \\
0
\end{array}\right)\right.
\end{aligned}
$$

From the last expression it is easy to see that we can get rid of the last $q_{j}-r_{j}^{*}$ columns of $H_{j}$. Thus, for every $j=1, \ldots, K$ we define a new matrix $H_{j}^{\star}$ by resetting all elements in $H_{j}$ to zero except for the elements in the first $r_{j}^{*}$ columns. Therefore, defining $\widetilde{\delta A_{j}}=\widehat{\delta A_{j}} H_{j}^{\star^{T}}$ we obtain matrices with rank $r_{j}^{*}$ that preserve the eigenvalue $\lambda_{\mathrm{RM}}$ and its right eigenvector $y$.

We still have to prove that $\left\|\widetilde{\delta A_{j}}\right\|_{F} \leq\left\|\delta A_{j}\right\|_{F}$; this is equivalent to proving that the spectral norm on each row of $\delta A_{j}$ is larger than the spectral norm of the same row in $\widetilde{\delta A_{j}}$. Without loss of generality let us investigate the spectral norms of the first row in each matrix. Let

$$
\left(\widehat{\delta A_{j}}\right)_{(1)}=\left[\begin{array}{lll}
\beta_{1} & \cdots & \beta_{q_{j}}
\end{array}\right]
$$

be the first row in $\widehat{\delta A_{j}}$ and the matrices $H_{j}^{T}$ and $H_{j}^{\star^{T}}$ defined as follows

$$
H_{j}^{T}=\left[\begin{array}{lllll}
p_{1}^{T} & p_{2}^{T} & p_{3}^{T} & \ldots & p_{q_{j}}^{T}
\end{array}\right]^{T}, \quad H_{j}^{\star^{T}}=\left[\begin{array}{llllll}
p_{1}^{T} & \ldots & p_{r_{j}^{*}}^{T} & 0 & \ldots & 0
\end{array}\right]^{T},
$$

with $p_{i} \in \mathbb{R}^{q_{j}}, i=1, \ldots, q_{j}$ orthonormal vectors.

Thus

$$
\begin{aligned}
& \left(\delta A_{j}\right)_{(1)}=\left(\widehat{\delta A_{j}}\right)_{(1)} H_{j}^{T}=\beta_{1} p_{1}^{T}+\cdots+\beta_{q_{j}} p_{q_{j}}^{T}, \\
& \left(\widehat{\delta A_{j}}\right)_{(1)}=\left(\widehat{\delta A_{j}}\right)_{(1)} H_{j}^{\star^{T}}=\beta_{1} p_{1}^{T}+\cdots+\beta_{r_{j}^{*}} p_{r_{j}^{*}}^{T} .
\end{aligned}
$$

Since $p_{1}, \ldots, p_{q_{j}}$ are orthonormal, it follows that

$$
\left\|\left(\widetilde{\delta A_{j}}\right)_{(1)}\right\|_{2} \leq\left\|\left(\delta A_{j}\right)_{(1)}\right\|_{2} .
$$


(ii) For each $j=1, \ldots, m$, let us indicate with $\left(\delta A_{j}\right)_{s, t}$ the coefficient in position $(s, t)$ of matrix $\delta A_{j}$, and with $B_{i, j}^{(s)}, C_{i, j(t)}$ respectively the s-th column of $B_{i, j}$ and the t-th row of $C_{i, j}$, for $s=1, \ldots, p_{j}, t=1, \ldots, q_{j}$. From Lemma 3.1, using some algebra manipulations we obtain that

$$
\begin{aligned}
\frac{\partial \mathfrak{R}\left(\lambda_{\mathrm{RM}}\right)}{\partial \delta A_{j}} & =\left(\frac{\partial \mathfrak{R}\left(\lambda_{\mathrm{RM}}\right)}{\partial\left(\delta A_{j}\right)_{s, t}}\right)_{\substack{s=1, \ldots, p_{j} \\
t=1, \ldots, q_{j}}}=-\frac{1}{\xi} \sum_{i=0}^{m} \mathfrak{R}\left(x^{*} B_{i, j}^{(s)} C_{i, j(t)} y e^{-\lambda_{\mathrm{RM}}\left(\tau_{i}+\delta \tau_{i}\right)}\right)_{\substack{s=1, \ldots, p_{j} \\
t=1, \ldots, q_{j}}}= \\
& -\frac{1}{\xi} \sum_{i=0}^{m}\left(B_{i, j}^{(s) T} \mathfrak{R}\left(x y^{*} e^{-\lambda_{\mathrm{RM}}\left(\tau_{i}+\delta \tau_{i}\right)}\right) C_{i, j(t)}^{T}\right)_{\substack{s=1, \ldots, p_{j} \\
t=1, \ldots, q_{j}}}=-\frac{1}{\xi} \sum_{i=0}^{m} B_{i, j}^{T} X \Gamma_{i} Y^{T} C_{i, j}^{T} \neq 0,
\end{aligned}
$$

where the last step stems from the hypothesis. As the optimal $\delta A_{j}$ must be $\varepsilon$-bounded, we impose the following constraints

$$
g_{j}:=\sum_{\substack{s=1, \ldots, p_{j} \\ t=1, \ldots, q_{j}}}\left(\delta A_{j}\right)_{s, t}^{2}-\varepsilon^{2} \leq 0, \quad j=1, \ldots, K .
$$

Since $\mathfrak{R}\left(\lambda_{\mathrm{RM}}\right)$ is a global maximum, from the theory of Lagrange multipliers we have that

$$
\frac{\partial \Re\left(\lambda_{\mathrm{RM}}\right)}{\partial \delta A_{j}}-2 \mu_{j} \delta A_{j}=0, \quad j=1, \ldots, K
$$

where $\mu_{j} \geq 0$ are the multipliers associated with the $g_{j}$ inequality constraints. Since we proved that $\frac{\partial \Re\left(\lambda_{\mathrm{RM}}\right)}{\partial \delta A_{j}}$ is nonzero, then $\mu_{j}$ is positive and $g_{j}$ is an active constraint; thus $\delta A_{j}$ is a positive multiple of $\frac{\partial \Re\left(\lambda_{\mathrm{RM}}\right)}{\partial \delta A_{j}}$ and in particular $\left\|\delta A_{j}\right\|_{F}=\varepsilon$ for $j=1, \ldots, K$, from which we derive the thesis.

(iii) The same reasoning used in point (ii) can be applied here; by hypothesis we have that

$$
\frac{\partial \mathfrak{R}\left(\lambda_{\mathrm{RM}}\right)}{\partial \delta \tau_{i}} \neq 0
$$

thus the constraint on the size of $\delta \tau_{i}$ must be active. As a consequence, the optimal $\delta \tau_{i}$ is such that $v_{i}\left|\delta \tau_{i}\right|=\varepsilon$.

The first statement of the theorem demonstrates that optimal perturbations of system matrices always have low-rank; as a consequence, the search space of optimization problem (3.2) can be reduced, and the new optimization problem that we want to solve reads as follows

$$
\begin{aligned}
\max & \Re \lambda(\Delta) \\
\text { s.t. } & \Delta \in \mathcal{S}:=\mathcal{S}_{F} \times \mathcal{S}_{\tau},
\end{aligned}
$$

where

$$
\begin{aligned}
\mathcal{S}_{F} & :=\left\{\Delta A \in \mathbb{R}^{*}: \operatorname{rank}\left(\delta A_{j}\right) \leq \rho_{j}^{*}=\min \left(2 k_{j}, p_{j}, q_{j}\right),\left\|\delta A_{j}\right\|_{F} \leq \varepsilon, j=1, \ldots, K\right\}, \\
\mathcal{S}_{\tau} & :=\prod_{i=1}^{m}\left[-\frac{\varepsilon}{v_{i}},+\frac{\varepsilon}{v_{i}}\right] .
\end{aligned}
$$

By definition, each point in $\mathcal{S}$ defines a perturbation of the nominal DEP. We want to construct a continuous path in $\mathcal{S}$ along which the spectral abscissa $\lambda$ is monotonically increasing. Hence, we consider perturbations $(\Delta A, \Delta \tau) \in \mathcal{S}$ as continuously depending on a parameter $t$ (as well as the corresponding rightmost eigenvalue $\lambda(t))$, and we use the decomposition

$$
\left\{\begin{array}{lll}
\delta A_{j}(t)=-\varepsilon U_{j}(t) Q_{j}(t) V_{j}(t)^{T}, & & t \in \mathbb{R}^{+}, j=1, \ldots, K, \\
\delta \tau_{i}(t) & =\frac{\varepsilon}{v_{i}} q_{i}(t), & t \in \mathbb{R}^{+}, i=1, \ldots, m,
\end{array}\right.
$$


where the following properties are satisfied

$$
\begin{cases}U_{j}(t)^{T} \dot{U}_{j}(t)=0, & \forall t \geq 0 \\ V_{j}(t)^{T} \dot{V}_{j}(t)=0, & \forall t \geq 0 \\ \left\|Q_{j}(t)\right\|_{F} \leq 1, & \forall t \geq 0 \\ \left|q_{i}(t)\right| \leq 1, & \forall t \geq 0\end{cases}
$$

with $U_{j}(t) \in \mathbb{R}^{p_{j} \times \rho_{j}^{*}}, V_{j}(t) \in \mathbb{R}^{q_{j} \times \rho_{j}^{*}}, Q_{j}(t) \in \mathbb{R}^{\rho_{j}^{*} \times \rho_{j}^{*}}$ for $j=1, \ldots, K$, and $q_{i}(t) \in \mathbb{R}$ for $i=1, \ldots, m$. We refer to [13] for more information with respect to this kind of parameterization.

Indicating with $\langle A, B\rangle=\operatorname{Trace}\left(A^{T} B\right)$ the Frobenius inner product of two matrices, it is easy to prove that the quantities satisfying properties (3.9) can be characterized, without losing of generality, as the solutions of the following differential equations

$$
\begin{aligned}
\dot{U}_{j}(t) & =\left(I_{n}-U_{j}(t) U_{j}(t)^{T}\right) R_{j}(t), \\
\dot{V}_{j}(t) & =\left(I_{n}-V_{j}(t) V_{j}(t)^{T}\right) S_{j}(t), \\
\dot{Q}_{j}(t) & = \begin{cases}M_{j}(t)-\left\langle M_{j}(t), Q_{j}(t)\right\rangle Q_{j}(t), & \text { if }\left\|Q_{j}(t)\right\|_{F}=1,\left\langle M_{j}(t), Q_{j}(t)\right\rangle>0 \\
M_{j}(t), & \text { otherwise, } \\
0 & \text { if }\left|q_{i}(t)\right|=1, r_{i}(t) q_{i}(t)>0 \\
\dot{q}_{i}(t) & \text { otherwise, }\end{cases}
\end{aligned}
$$

where we have introduced the arbitrary matrices $R_{j}(t) \in \mathbb{R}^{p_{j} \times \rho_{j}^{*}}, S_{j}(t) \in \mathbb{R}^{q_{j} \times \rho_{j}^{*}}, M_{j}(t) \in \mathbb{R}^{\rho_{j}^{*} \times \rho_{j}^{*}}$ for $j=$ $1, \ldots, K$ and the arbitrary functions $r_{i}(t)$ for $i=1, \ldots, m$.

Now, we want to identify matrices $R_{j}(t), S_{j}(t), M_{j}(t)$ and functions $q_{i}(t)$ such that $\dot{\mathfrak{R}}(\lambda(t)) \geq 0$. This will enable us to individuate, for each $t$, a direction in the space of perturbations $\mathcal{S}$ where the real part of the rightmost eigenvalue $\lambda(t)$ is monotonically increasing. From Lemma 3.1, the derivative of an eigenvalue $\lambda$ w.r.t. the parameter $t$ reads

$$
\frac{d \lambda(t)}{d t}=\frac{1}{\xi}\left\{x^{*}\left(\sum_{i=0}^{m} \sum_{j=1}^{K}-B_{i, j} \delta \dot{A}_{j}(t) C_{i, j} e^{-\lambda\left(\tau_{i}+\delta \tau_{i}(t)\right)}+\sum_{i=0}^{m}\left(A_{i}+\sum_{j=1}^{K} B_{i, j} \delta A_{j}(t) C_{i, j}\right) \lambda \delta \dot{\tau}_{i}(t) e^{-\lambda\left(\tau_{i}+\delta \tau_{i}(t)\right)}\right) y\right\},
$$

with $\xi$ defined as in Theorem 3.2. Thus, substituting equations (3.10) and considering the easy case where $\dot{Q}_{j}=M_{j}$ and $\dot{q}_{i}=r_{i}$, we have

$$
\begin{aligned}
& \mathfrak{R}(\dot{\lambda})=\sum_{i=0}^{m} \sum_{j=1}^{K} \frac{\varepsilon}{\xi} \mathfrak{R}\left(x^{*} B_{i, j} \dot{U}_{j} Q_{j} V_{j}^{T} C_{i, j} e^{-\lambda\left(\tau_{i}+\delta \tau_{i}\right)} y\right)+\sum_{i=0}^{m} \sum_{j=1}^{K} \frac{\varepsilon}{\xi} \mathfrak{R}\left(x^{*} B_{i, j} U_{j} \dot{Q}_{j} V_{j}^{T} C_{i, j} e^{-\lambda\left(\tau_{i}+\delta \tau_{i}\right)} y\right) \\
& +\sum_{i=0}^{m} \sum_{j=1}^{K} \frac{\varepsilon}{\xi} \mathfrak{R}\left(x^{*} B_{i, j} U_{j} Q_{j} \dot{V}_{j}^{T} C_{i, j} e^{-\lambda\left(\tau_{i}+\delta \tau_{i}\right)} y\right)+\sum_{i=0}^{m} \frac{\varepsilon}{\xi v_{i}} \mathfrak{R}\left(x^{*}\left(A_{i}+\sum_{j=1}^{K} B_{i, j} \delta A_{j} C_{i, j}\right) \lambda \dot{q}_{i} e^{-\lambda\left(\tau_{i}+\delta \tau_{i}\right)} y\right) \\
& =\sum_{i=0}^{m} \sum_{j=1}^{K} \frac{\varepsilon}{\xi}\left\langle X, B_{i, j}\left(I_{n}-U_{j} U_{j}^{T}\right) R_{j} Q_{j} V_{j}^{T} C_{i, j} Y \Gamma_{i}^{T}\right\rangle+\sum_{i=0}^{m} \sum_{j=1}^{K} \frac{\varepsilon}{\xi}\left\langle X, B_{i, j} U_{j} M_{j} V_{j}^{T} C_{i, j} Y \Gamma_{i}^{T}\right\rangle \\
& +\sum_{i=0}^{m} \sum_{j=1}^{K} \frac{\varepsilon}{\xi}\left\langle X, B_{i, j} U_{j} Q_{j} S_{j}^{T}\left(I_{n}-V_{j} V_{j}^{T}\right) C_{i, j} Y \Gamma_{i}^{T}\right\rangle+\sum_{i=0}^{m} \frac{\varepsilon}{\xi v_{i}} \underbrace{\Re\left(x^{*}\left(A_{i}+\sum_{j=1}^{K} B_{i, j} \delta A_{j} C_{i, j}\right) \lambda e^{-\lambda\left(\tau_{i}+\delta \tau_{i}\right)} y\right)}_{:=\beta_{i}} \dot{q}_{i} \\
& =\sum_{j=1}^{K} \frac{\varepsilon}{\xi}\left\langle\sum_{i=0}^{m} B_{i, j}^{T} X \Gamma_{i} Y^{T} C_{i, j}^{T} V_{j} Q_{j}^{T},\left(I_{n}-U_{j} U_{j}^{T}\right) R_{j}\right\rangle+\sum_{j=1}^{K} \frac{\varepsilon}{\xi}\left\langle\sum_{i=0}^{m} U_{j}^{T} B_{i, j}^{T} X \Gamma_{i} Y^{T} C_{i, j}^{T} V_{j}, M_{j}\right\rangle \\
& +\sum_{j=1}^{K} \frac{\varepsilon}{\xi}\left\langle S_{i},\left(I_{n}-V_{j} V_{j}^{T}\right) \sum_{i=0}^{m} C_{i, j} Y \Gamma_{i}^{T} X^{T} B_{i, j} U_{j} Q_{j}\right\rangle+\sum_{i=0}^{m} \frac{\varepsilon}{\xi v_{i}} \beta_{i} \dot{q}_{i},
\end{aligned}
$$


where parameter $t$ has been neglected to simplify the notation. Therefore, since $\left(I_{n}-U_{j} U_{j}^{T}\right),\left(I_{n}-V_{j} V_{j}^{T}\right)$ are positive semi-definite, the steepest ascent is guaranteed by the following choices

$$
\begin{aligned}
R_{j} & =\sum_{i=0}^{m} B_{i, j}^{T} X \Gamma_{i} Y^{T} C_{i, j}^{T} V_{j} Q_{j}^{T}, & S_{j} & =\sum_{i=0}^{m} C_{i, j} Y \Gamma_{i}^{T} X^{T} B_{i, j} U_{j} Q_{j}, \\
M_{j} & =U_{j}^{T} \sum_{i=0}^{m} B_{i, j}^{T} X \Gamma_{i} Y^{T} C_{i, j}^{T} V_{j}, & r_{i} & =\beta_{i} .
\end{aligned}
$$

Note that the other cases for differential equations (3.10) give the same expressions.

As a consequence of the definition of differential equations (3.10) with choices (3.11), at each fixed $t$ the solutions $U_{j}(t), V_{j}(t), Q_{j}(t), q_{i}(t)$ define a new perturbed DEP such that $\mathfrak{R}(\lambda(t+\eta))>\mathfrak{R}(\lambda(t))$ for $\eta>0$ sufficiently small, where $\lambda(t)$ is the rightmost eigenvalue of the problem. In our algorithm, we move along the trajectory of these solutions using a discretization of the differential equations (3.10). At each iteration $k$, we first perform a forward Euler method with adaptive stepsize together with a projection of the new quantities onto the space of perturbations $\mathcal{S}$, in order to satisfy the desired properties listed in (3.9). Statement (ii) of Theorem 3.2 is used for the initialization of the algorithm, which can be interpreted as the first step of the fixed-point iteration suggested in equation (3.4). Delay perturbations are simply initialized following the derivative computed in Equation (3.5). We refer the reader interested in a detailed illustration of the method to Algorithm 1 in [1].

The algorithm converges when the norm of the gradient of the spectral abscissa is under a given tolerance: clearly this condition is also satisfied on local maxima, namely locally but not globally rightmost points of the pseudospectrum. In order to avoid a convergence to such a point, restarting the method from different eigenvalues located on the right part of the original spectrum has proved to be effective. The DEPs in the algorithm are solved using the algorithm and the software described in [16].

\section{Smoothness properties and optimization of the pseudospectral ab- scissa}

In the previous section we presented an exact method for assessing robust stability when the system is subject to perturbations as in (2.1). In this section we make the leap to the design of robustly stabilizing controllers.

The robust stabilization approach is based on minimizing the pseudospectral abscissa as a function of controller parameters contained in vector $p \in \mathbb{R}^{n_{p}}$. Considering again Example 2.1, the vector of controller parameters is $p=K^{T}$ if only gain is optimized, and $p=\left[K^{T} ; \tau\right]$ if both gain and delay are used as optimization variables. The latter case will be considered again in the experiment described in Example 5.1 of the next section.

From now on, we take again into account the dependence of the perturbed DEP (2.2) and of the pseudospectral abscissa on the set of parameters $p$, as follows

$$
\begin{aligned}
\alpha_{\varepsilon}(p): \mathbb{R}^{n_{p}} & \longrightarrow \mathbb{R} \\
p & \longrightarrow \alpha_{\varepsilon}(M(\lambda, p)),
\end{aligned}
$$

It is important to highlight that the maximum size of the uncertainties is not depending on the set of parameters $p$, although these parameters may admit an uncertainty too.

Let us first briefly summarize the pseudospectral abscissa smoothness properties: as previously mentioned, Assumptions 2.1 and 2.2 guarantee that the spectral abscissa is continuous with respect to matrix coefficients and delay terms, and also w.r.t. their potential perturbations. Then, following from the properties of the maximum function, the pseudospectral abscissa is also continuous but not everywhere differentiable; typically it is differentiable almost everywhere, whereas points of non-smoothness are in most cases generated by the presence of the maximum function and are characterized by switching of the component of pseudospectrum which contains the globally rightmost eigenvalue. For this reason, the function is often non-differentiable in its local minima. Furthermore, the pseudospectral abscissa is in general non-Lipschitz and non-convex.

We would like to minimize $\alpha_{\varepsilon}$ w.r.t. to the set of controller or design parameters: to this purpose, we use the HANSO algorithm ([23]), which has been proved to efficiently converge to local minima of nonsmooth, nonconvex functions: example of its applications in this field can be found in [16] and [9], where respectively 
the spectral abscissa and the robust $H_{\infty}$ norm of a system of DDAEs are optimized. The application of the HANSO software only requires the availability of a routine to evaluate the objective function and its gradient, whenever the objective function is differentiable.

Next theorem provides the explicit expression of the derivative of $\alpha_{\varepsilon}$ w.r.t. to the design or controller parameters $p$, whenever this derivative exists.

Theorem 4.1. Let $\left(\lambda_{\mathrm{RM}}(p), x(p), y(p)\right)$ be respectively the globally rightmost point of $\Lambda_{\varepsilon}$ and its left and right normalized eigenvectors; for each set of parameter $p \in \mathbb{R}^{n_{p}}$ let us define $\widetilde{\Delta A}(p)=\left(\widetilde{\delta A_{1}}(p), \ldots, \widetilde{\delta A_{K}}(p)\right)$ and $\widetilde{\Delta \tau}(p)=\left(\widetilde{\delta \tau_{1}}(p), \ldots, \widetilde{\delta \tau_{m}}(p)\right)$ as the optimal matrix perturbations and optimal delay perturbations functions such that $\lambda_{\mathrm{RM}}(p)$ is simple; then denoting

$$
\zeta:=-x^{*}\left(E+\sum_{i=0}^{m}\left(A_{i}+\sum_{j=1}^{K} B_{i, j} \widetilde{\delta A_{j}} C_{i, j}\right)\left(\tau_{i}+\widetilde{\delta \tau_{i}}\right) e^{-\lambda_{\mathrm{RM}}\left(\tau_{i}+\widetilde{\delta \tau_{i}}\right)}\right) y
$$

we can express the derivative of the pseudospectral abscissa w.r.t each parameter $p_{j}$ as follows

$$
\frac{\partial \alpha_{\varepsilon}}{\partial p_{j}}=\frac{\partial \mathfrak{R}\left(\lambda_{\mathrm{RM}}\right)}{\partial p_{j}}=\frac{1}{\zeta} \mathfrak{R}\left[x^{*}\left(-\sum_{i=0}^{m} \frac{\partial A_{i}}{\partial p_{j}} e^{-\lambda_{\mathrm{RM}}\left(\tau_{i}+\widetilde{\delta \tau_{i}}\right)}+\sum_{i=0}^{m}\left(A_{i}+\sum_{j=1}^{K} B_{i, j} \widetilde{\delta A_{j}} C_{i, j}\right) e^{-\lambda_{\mathrm{RM}}\left(\tau_{i}+\widetilde{\delta \tau_{i}}\right)} \lambda_{\mathrm{RM}} \frac{\partial \tau_{i}}{\partial p_{j}}\right) y\right]
$$

where we omit the dependence on $p$ to simplify the notation and again assumed $\tau_{0}=0$.

Observe that the values of the optimal perturbations $\widetilde{\delta A_{i}}, \widetilde{\delta \tau_{i}}$ does depend on the set of design or controller parameters $p$ : indeed, although the maximum size of the optimal perturbations is prescribed, the optimal perturbations might assume different values in the parameter space. However, due to the optimality conditions, their derivative w.r.t. $p$ does not affect the derivative of the pseudospectral abscissa.

The main scope of our work is to tune parameters in order to have a negative pseudospectral abscissa. For this reason, convergence to a local but not global minimum of the function does not represent an issue; even more, from a practical point of view, any negative value for the pseudospectral abscissa ensures a robust stability of our system, regardless of the convergence of the algorithm to a local minimum.

HANSO algorithm is intended for unconstrained optimization; however, we might need to force our controller to stay in some region of the parameter space, e.g. we constrain the delay terms to have positive values. For this reason, we also include some penalties in our cost function, as we will explain case by case in the following section. In general, the penalties we introduce are continuous but non differentiable functions. It is worth to remark that HANSO method can cope with non-differentiability; moreover, the pseudospectral abscissa function is likely to be non-differentiable in its minima, so we do not add any non-smoothness to the cost function.

\section{Numerical experiments}

Here we report three different numerical experiments: the first two are mathematical examples respectively from [5] and [28] and prove the effectiveness of the method and the need of the barrier function to constrain delays to be positive. The third example is an application to the cutting machine already introduced in Example 2.2 .

Example 5.1. We consider the system introduced in Example 2.1, where

$$
A=\left[\begin{array}{rrr}
-0.08 & -0.03 & 0.2 \\
0.2 & -0.04 & -0.005 \\
-0.06 & 0.2 & -0.07
\end{array}\right], \quad B=\left[\begin{array}{r}
-0.1 \\
-0.2 \\
0.1
\end{array}\right], \quad C=I_{3}
$$

For a nominal delay $\tau=1$ this system is unstable, therefore we introduce a static feedback $u(t)=K y(t)$ and we consider $\tau$ itself as an optimization variable: thus, the vector of controller parameters is $p=\left[K^{T} ; \tau\right]$. As in Example 2.1, we include uncertainties $\delta A, \delta B$ on the matrix coefficients, while $C$ remains unperturbed; we also assume an uncertainty $\delta \tau$ on the delay $\tau$ satisfying $|\delta \tau| \leq 0.5$. Thus we set $\delta A_{1}=\delta A, \delta A_{2}=\delta B$,

$$
\varepsilon=0.05, B_{0,1}=\|A\|_{F}\left[\begin{array}{l}
I \\
0 \\
0
\end{array}\right], B_{1,2}=\|B\|_{F}\left[\begin{array}{l}
I \\
0 \\
0
\end{array}\right], C_{0,1}=\left[\begin{array}{l}
I \\
0 \\
0
\end{array}\right]^{T}, C_{1,2}=\left[\begin{array}{l}
0 \\
0 \\
I
\end{array}\right]^{T}, v_{1}=\frac{\varepsilon}{0.5} .
$$


Table 1: The table shows the spectral abscissa $\alpha$ and the pseudospectral abscissa $\alpha_{\varepsilon}$ in the uncontrolled system $\left(p=\left[\begin{array}{llll}0 & 0 & 0 & 1\end{array}\right]\right)$, and in the systems with controllers $p^{*}$ and $p_{\varepsilon}^{*}$.

\begin{tabular}{c|cc}
\hline & $\alpha$ & $\alpha_{\varepsilon}$ \\
\hline Uncontrolled & $+1.0806 \mathrm{e}-01$ & $+1.2659 \mathrm{e}-01$ \\
$p^{*}$ & $-5.2374 \mathrm{e}-01$ & $-1.4720 \mathrm{e}-01$ \\
$p_{\varepsilon}^{*}$ & $-2.5966 \mathrm{e}-01$ & $-1.7420 \mathrm{e}-01$ \\
\hline
\end{tabular}

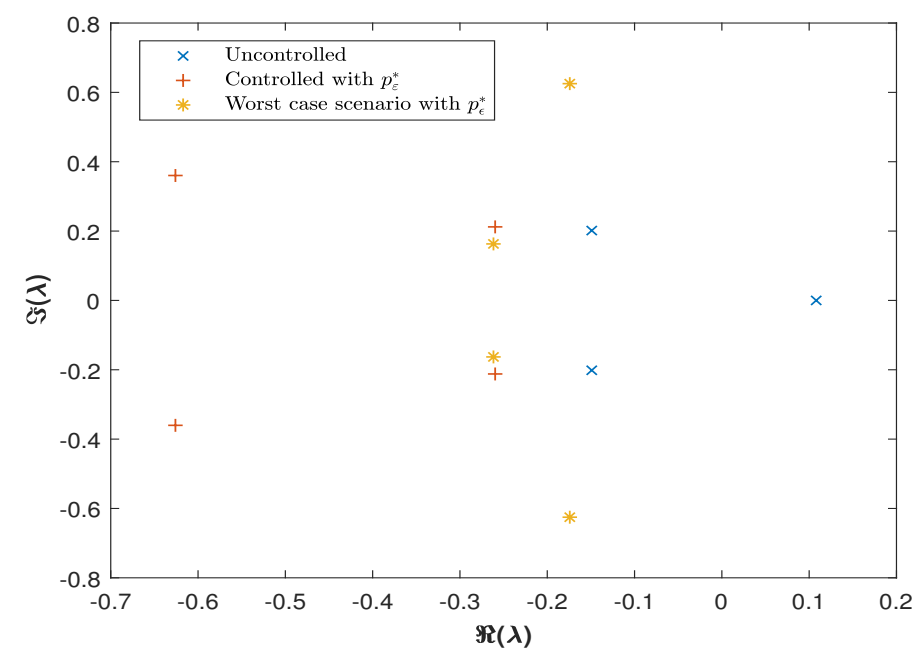

Figure 1: The figure shows the rightmost eigenvalues for $K=0$, for $K=K_{\varepsilon}^{*}$ and the worst-case scenario for $K=K_{\varepsilon}^{*}$.

With these choices, we allow a maximal relative error of $5 \%$ on matrices $A, B$. Note that a barrier function is needed to prevent the delay $\tau$ from assuming negative values: since the uncertainty $\delta \tau$ such that $|\delta \tau| \leq 0.5$ is also present, we constrain nominal value $\tau$ to be larger than 0.5 adding a continuous penalty to our cost function as follows

$$
\begin{aligned}
\alpha_{\varepsilon}: \mathbb{R}^{4} & \longrightarrow \mathbb{R} \\
(K, \tau) & \longrightarrow \begin{cases}\alpha_{\varepsilon}(M(\lambda, K, \tau)), & \text { if } \tau \geq 0.5 \\
\alpha_{\varepsilon}(M(\lambda, K, 0.5))+10(0.5-\tau), & \text { if } \tau<0.5\end{cases}
\end{aligned}
$$

In Table 1 we report the values of the spectral abscissa $\alpha$ and of the pseudospectral abscissa $\alpha_{\varepsilon}$ in three different cases: in the first case the system is uncontrolled $\left(p_{0}=[0 ; 0 ; 0 ; 1]\right)$; in the second case the controller $p^{*}=[1.4784 ; 2.6415 ; 2.3611 ; 1.1351]$ is a minimizer for the spectral abscissa function $\alpha$ (of course w.r.t. the same controller variables $p)$; in the third case the optimal controller $p_{\varepsilon}^{*}=[2.0671 ; 3.5925 ; 3.7166 ; 0.8855]$ is a minimizer for the pseudospectral abscissa $\alpha_{\varepsilon}$ in (5.1). It is worth remarking that $\alpha_{\varepsilon}\left(p_{\varepsilon}^{*}\right)<0$ : this means that our optimization process provided an optimal controller that guarantees stability against system's uncertainties. We also observe that $\alpha_{\varepsilon}\left(p_{\varepsilon}^{*}\right)<\alpha_{\varepsilon}\left(p^{*}\right)$ and that $\alpha\left(p_{\varepsilon}^{*}\right)>\alpha\left(p^{*}\right)$ : this demonstrates the considerably different behaviors of functions $\alpha$ and $\alpha_{\varepsilon}$ and justifies our optimization approach for the robust stabilization. Finally, in Figure 1 we compare the rightmost eigenvalues in the uncontrolled system, in the system controlled with $p=p_{\varepsilon}^{*}$ and in the worst-case scenario for $p=p_{\varepsilon}^{*}$, where the real part of the globally rightmost eigenvalue is the pseudospectral abscissa.

Example 5.2. In this example we consider system

$$
\dot{x}(t)=A_{0} x(t)+A_{1} x\left(t-\tau_{1}\right)+A_{2} x\left(t-\tau_{2}\right),
$$

with

$$
A_{0}=\left[\begin{array}{ccc}
-1 & 13.5 & -1 \\
-3 & -1 & -2 \\
-2 & -1 & -4
\end{array}\right], A_{1}=\left[\begin{array}{ccc}
-5.9 & 0 & 0 \\
2 & 0 & 0 \\
2 & 0 & 0
\end{array}\right], A_{2}=\left[\begin{array}{ccc}
0 & 7.1 & -70.3 \\
0 & -1 & 5 \\
0 & 0 & 6
\end{array}\right]
$$




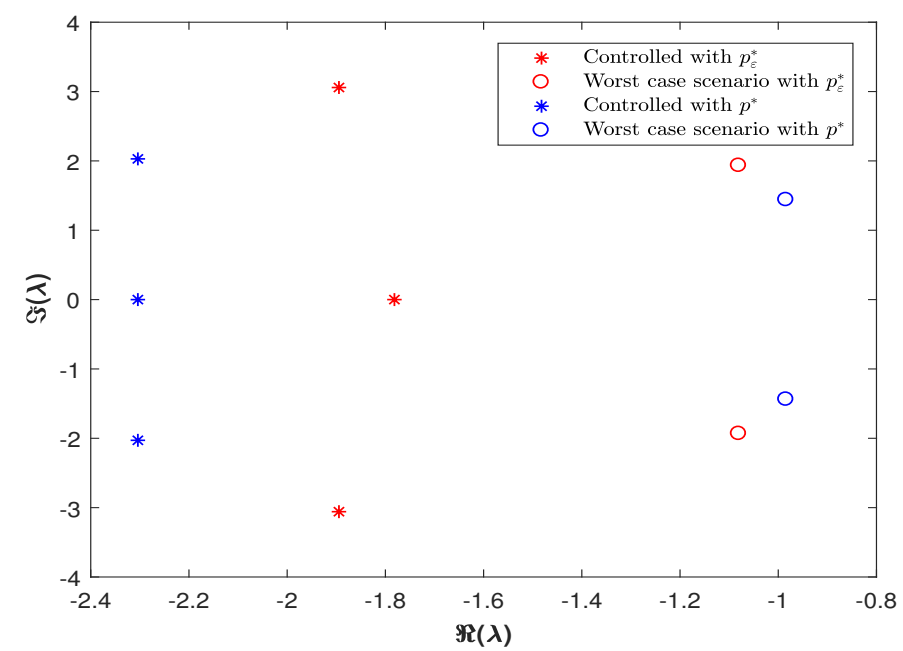

Figure 2: The figure shows the rightmost eigenvalues of the spectra obtained for the controllers $p^{*}$ and $p_{\varepsilon}^{*}$, and the corresponding worst-case scenarios.

whose stability w.r.t. the delay terms $\tau_{1}$ and $\tau_{2}$ is examined in [5]. In this example, time delays are considered unperturbed, and we assume an uncertainty $\delta A_{1}$ on matrix $A_{0}$ and an uncertainty $\delta A_{2}$ affecting the nonzero part of matrix $A_{1}$. Thus, we define

$$
B_{0,1}=\left\|A_{0}\right\|_{F} I_{3}, \quad C_{0,1}=I_{3}, \quad B_{1,2}=\left\|A_{1}\right\|_{F} I_{3}, \quad C_{1,2}=\left[\begin{array}{lll}
1 & 0 & 0
\end{array}\right],
$$

where $I_{3}$ is the identity matrix with dimension 3 , and we set $\varepsilon=0.005$. We now consider the minimization of the pseudospectral abscissa, where the delays $\tau_{1}$ and $\tau_{2}$ are assumed as controller parameters. Again, we include some continuous penalties in our cost function in order to prevent the delay terms from being negative

$$
\begin{aligned}
\alpha_{\varepsilon}: \quad \mathbb{R}^{2} & \longrightarrow \mathbb{R} \\
\left(\tau_{1}, \tau_{2}\right) & \longrightarrow \begin{cases}\alpha_{\varepsilon}\left(M\left(\lambda, \tau_{1}, \tau_{2}\right)\right), & \text { if } \tau_{1} \geq 0, \tau_{2} \geq 0 \\
\alpha_{\varepsilon}\left(M\left(\lambda, 0, \tau_{2}\right)\right)-100 \tau_{1}, & \text { if } \tau_{1}<0, \tau_{2} \geq 0 \\
\alpha_{\varepsilon}\left(M\left(\lambda, \tau_{1}, 0\right)\right)-100 \tau_{2}, & \text { if } \tau_{1} \geq 0, \tau_{2}<0 \\
\alpha_{\varepsilon}(M(\lambda, 0,0))-100 \tau_{1}-100 \tau_{2}, & \text { if } \tau_{1}<0, \tau_{2}<0\end{cases}
\end{aligned}
$$

Optimizing function $\alpha_{\varepsilon}$ as defined in (5.2), a minimizer is found in $p_{\varepsilon}^{*}=[1.8955 e-02 ; 1.5599 e-02]$, whereas the minimization of the spectral abscissa $\alpha$ converged to a point $p^{*}=[3.5490 e-05 ; 1.3920 e-02]$. The controllers are close to the negative half-planes for both variables $\tau_{1}, \tau_{2}$, however the configuration $\left(\tau_{1}, \tau_{2}\right)=(0,0)$ is not a minimizer. In Figure 2 we compare the spectral abscissae and the worst-case scenarios for both controllers $p^{*}$ and $p_{\varepsilon}^{*}$ : the observations made for the previous example still hold, as $\alpha\left(p^{*}\right)<\alpha\left(p_{\varepsilon}^{*}\right)$ and $\alpha_{\varepsilon}\left(p_{\varepsilon}^{*}\right)<\alpha_{\varepsilon}\left(p^{*}\right)$.

Example 5.3. We conclude this section analysing the concrete example of a cutting machine performing a turning operation. We thus examine again the system introduced in Example 2.2, but this time we only consider an uncertainty $\delta k=\delta A_{1}$ on the cutting coefficient $k$ and an uncertainty $\delta \tau$ on the delay term; in particular, we impose $|\delta k| \leq 1.5 * 10^{6}$ and $|\delta \tau| \leq 0.0015$. Therefore, we assume

$$
B_{1,1}=-B_{2,1}=\left[\begin{array}{c}
0 \\
\frac{1}{m}
\end{array}\right], \quad C_{1,1}=C_{2,1}=1.5 * 10^{7}\left[\begin{array}{ll}
1 & 0
\end{array}\right] .
$$

and we set $\varepsilon=0.1, v=0.015^{-1}$, with $v$ the weight associated with perturbation $\delta \tau$. In Figure 3 the blue line is the level set equal to 0 of the spectral abscissa, therefore the region below is the stability region in the $k$ - $\tau$ space for system (2.5); the red line is the level set equal to 0 of the pseudospectral abscissa $\alpha_{\varepsilon}$. The rectangular blocks represent all the perturbations $(\delta k, \delta \tau)$ within a distance $\varepsilon$ from the point marked with a red asterisk. By definition, for each point whose $\alpha_{\varepsilon}=0$ we can build a $\Delta=(\delta k, \delta \tau)$ with $\|\Delta\|_{\text {glob }}=\varepsilon$ such that the perturbed system is unstable. Finally, in Figure 3 we have represented the iterations of the minimization process of the 


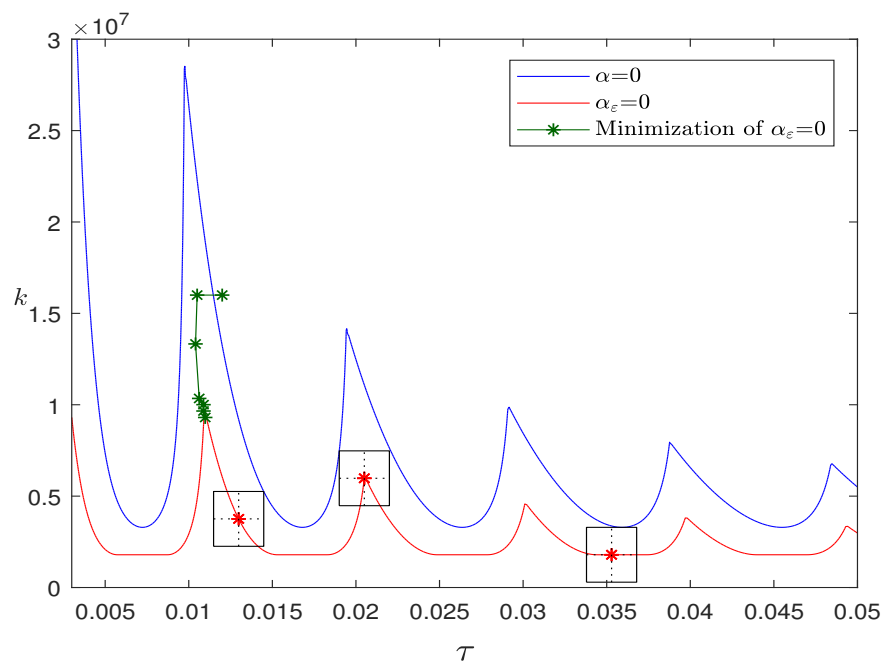

Figure 3: Red and blue lines represent respectively the level sets for $\alpha_{\varepsilon}=0$ and for $\alpha=0$. The iterations of the optimization of the pseudospectral abscissa are indicated by the green points.

following cost function, where penalties are added to constrain the cutting force coefficient $k \geq 2 * 10^{6}:=k_{\min }$ and the delay term $\tau \geq 0.005:=\tau_{\min }$

$$
\begin{aligned}
\alpha_{\varepsilon}: \quad \mathbb{R}^{2} & \longrightarrow \mathbb{R} \\
(k, \tau) & \longrightarrow \begin{cases}\alpha_{\varepsilon}(M(\lambda, k, \tau)), & \text { if } \tau_{1} \geq 0, \tau_{2} \geq 0, \\
\alpha_{\varepsilon}\left(M\left(\lambda, k, \tau_{\min }\right)\right)-1000\left(\tau_{\min }-\tau\right), & \text { if } k \geq k_{\min }, \tau<\tau_{\min }, \\
\alpha_{\varepsilon}\left(M\left(\lambda, k_{\min }, \tau\right)\right)-1000\left(k_{\min }-k\right), & \text { if } k<k_{\min }, \tau \geq \tau_{\min }, \\
\alpha_{\varepsilon}\left(M\left(\lambda, k_{\min }, \tau_{\min }\right)\right)-1000\left(k_{\min }-k\right)-1000\left(\tau_{\min }-\tau\right), & \text { if } k<k_{\min }, \tau<\tau_{\min },\end{cases}
\end{aligned}
$$

Note from the graphical interpretation that the only minima of the cost function defined in (5.3) are generated by the introduction of the penalty on parameter $k$ : since we are mostly interested in a robustly stable configuration, in this case we stop the algorithm as soon as a negative value for $\alpha_{\varepsilon}$ is found.

\section{Concluding remarks}

In this chapter we illustrated a method to design a robust controller for a class of linear DDAEs affected by uncertainty on the system matrices and on the delay values. The method consists of the minimization of the pseudospectral abscissa w.r.t. some controller or design parameters: the optimization variables can either be matrix coefficients or delay values. The advantage of this method is evident: first, regarding the pseudospectral abscissa computation, a very broad class of perturbations is allowed on the system of DDAEs and the nonlinear structure of the associated delay eigenvalue problem is taken into account; as a consequence, the pseudospectral abscissa minimization allows to synthesize controllers for real-life applications, as it guarantees an asymptotically stable behavior of the solution despite the uncertainties affecting the system.

Applications of this method are envisaged in the design of static or dynamic fixed-order controllers that robustly stabilize uncertain system of DDEs. Moreover, since low-rank properties of optimal matrices perturbations are fully exploited and the algorithm only requires the computation of selected rightmost eigenvalues (for which fast iterative methods can be used), large-scale problems, which stem for instance from the discretization of PDEs with delay, can be dealt with as well.

\section{Acknowledgments}

This work was supported by the project C14/17/072 of the KU Leuven Research Council, by the project G0A5317N of the Research Foundation-Flanders (FWO - Vlaanderen), and by the project UCoCoS, funded by the European Unions Horizon 2020 research and innovation program under the Marie Sklodowska-Curie Grant Agreement No 675080 . 


\section{References}

[1] F. Borgioli and W. Michiels. Computing distance to instability for delay systems with uncertainties in the system matrices and in the delay terms (accepted). In Proceedings of the ECC, Limassol, Cyprus, 2018.

[2] F. Borgioli and W. Michiels. Robust stabilisation of linear time-delay systems with uncertainties in the system matrices and in the delay terms (accepted). In Proceedings of the 14th IFAC Workshop on Time-Delay Systems, Budapest, Hungary, 2018.

[3] E. Fridman. Stability of linear descriptor systems with delay: a Lyapunov-based approach. Journal of Mathematical Analysis and Applications, 273:24-44, 2002.

[4] E. Fridman. Tutorial on Lyapunov-based methods for time-delay systems. European Journal of Control, $20(6): 271$ - 283, 2014.

[5] E. Fridman and U. Shaked. $H_{\infty}$-control of linear state-delay descriptor systems: an LMI approach. Linear Algebra and its Applications, 351-352:271-302, 2002.

[6] E. Fridman and U. Shaked. An improved stabilization method for linear time-delay systems. IEEE transactions on automatic control, 47:1931-37, 2002.

[7] K. Gu, V.L. Kharitonov, and J. Chen. Stability of time-delay systems. Birkhauser, 2003.

[8] N. Guglielmi and C. Lubich. Low-rank dynamics for computing extremal points of real pseudospectra. SIAM Journal of Matrix Analysis and Applications, 34:40-66, 2013.

[9] Suat Gumussoy and Wim Michiels. Fixed-order strong h-infinity control of interconnected systems with time-delays. IFAC Proceedings Volumes, 44:12544 - 12549, 2011. 18th IFAC World Congress.

[10] G. Hu and E. J. Davison. Real stability radii of linear time-invariant time-delay systems. Systems E Control Letters, 50:209 - 219, 2003.

[11] T Insperger and G. Stépán. Stability of the milling process. Periodica Polytechnica Mechanical Engineering, 44(1):47-57, 2000.

[12] S. Jayaram, S.G. Kapoor, and R.E. DeVor. Analytical stability analysis of variable spindle speed machines. Journal of Manufacturing and Engineering, 122:391-397, 2000.

[13] O. Koch and C. Lubich. Dynamical low rank approximation. SIAM J. Matrix Anal. Appl., 29(2):434-454, 2007.

[14] M. Krstic. Delay compensation for nonlinear, adaptive and PDE systems. Birkhauser, 2007.

[15] A. Lewis and M.L. Overton. Nonsmooth optimization via BFGS. Available from http://cs.nyu.edu/overton/papers.html.

[16] W. Michiels. Spectrum based stability analysis and stabilization of systems described by delay differential algebraic equations. IET Control Theory and Applications, 5(16):1829-1842, 2011.

[17] W. Michiels, K. Engelborghs, P. Vansevenant, and D. Roose. The continuous pole placement method for delay equations. Automatica, 38(5):747-761, 2002 .

[18] W. Michiels, K. Green, T. Wagenknecht, and S.I. Niculescu. Pseudospectra and stability radii for analytic matrix functions with applications to time-delay systems. Linear Algebra and its Applications, 418(1):315-335, 2006.

[19] W. Michiels and N. Guglielmi. An iterative method for computing the pseudospectral abscissa for a class of nonlinear eigenvalue problems. SIAM Journal on Scientific Computing, 34(4):A2366-A2393, 2012.

[20] W. Michiels and S.I. Niculescu. On the delay sensitivity of smith predictors. Internation journal of systems science, 34:543-52, 2003.

[21] W. Michiels and S.I. Niculescu. Stability and stabilization of time-delay systems. An eigenvalue based approach. SIAM, 2007.

[22] S.I. Niculescu. Delay effects on stability. A robust control approach, volume 269 of Lecture Notes in Control and Information Sciences. Springer-Verlag, 2001.

[23] M. Overton. HANSO: a hybrid algorithm for nonsmooth optimization. Available from http://cs.nyu.edu/overton/software/hanso/, 2009

[24] Z.J. Palmor. Time-delay compensation - Smith predictor and its modifications. In S. Levine, editor, The Control Handbook. CRC and IEEE Press, New York, 1996.

[25] P. Pepe, I. Karafyllis, and Z.-P. Jiang. On the Liapunov-Krasovskii methodology for the ISS of systems described by coupled delay differential and difference equations. Automatica, 44(9):2266-2273, 2008.

[26] K. Schreiber. Nonlinear eigenvalue problems: Newton-type methods and nonlinear Rayleigh functionals. PhD thesis, TU Berlin, 2008.

[27] A. Seuret and K. H. Johansson. Stabilization of time-delay systems through linear differential equations using a descriptor representation. In Proceedings of the ECC, pages 4727-32, Budapest, Hungary, 2009.

[28] J. Vanbiervliet, K. Verheyden, W. Michiels, and S. Vandewalle. A nonsmooth optimization approach for the stabilization of linear time-delay systems. ESAIM: Control, Optimisation and Calcalus of Variations, 14(3):478-493, 2008. 